\title{
Um Estudo sobre a Contribuição de Ontologias para a Educação suportada por Tecnologias da Informação no Domínio da Saúde
}

\author{
Sidney Patrézio R. Campos', Cecilia N. A. P. Gomes ${ }^{2}$, Natasha C. Q. Lino ${ }^{1}$ \\ ${ }^{1}$ Programa de Pós-Graduação em Informática (PPGI), Universidade Federal da Paraíba \\ (UFPB), João Pessoa - PB - Brasil \\ ${ }^{2}$ Programa de Pós-Graduação em Modelos de Decisão e Saúde (PPGMDS), \\ Universidade Federal da Paraíba (UFPB), João Pessoa - PB - Brasil \\ sidneypatrezio@di.ufpb.br, netapegado@gmail.com, natasha@ci.ufpb.br
}

\begin{abstract}
Education supported by information technologies is growing and setting a new paradigm in this important human activity area. In this context, Health Informatics has been focusing in the application and development of diverse software, approaches and computational methods with educational purposes, in which, most of them use Ontologies, a technique of Knowledge Representation, that permit semantic modeling and description of concepts within a domain in the Health area. The main goal of this study is to identify the state of the art about how Ontologies contribute for education supported by Information Technology in the Health domain.
\end{abstract}

Resumo. A educação suportada por tecnologias da informação vem ganhando espaço e estabelecendo um novo paradigma nesta importante área da atividade humana. Neste contexto, a Informática em Saúde tem se dedicado a aplicação $e$ desenvolvimento de diferentes softwares, abordagens e métodos computacionais com propósitos educacionais, dos quais muitos utilizam ontologias, que são técnicas de Representação do Conhecimento, para a modelagem e descrição semântica de conceitos de um determinado domínio da área da saúde. O principal objetivo deste estudo é identificar o estado da arte sobre como ontologias podem contribuir para a educação suportada por tecnologias da informação no domínio da saúde.

\section{Introdução}

Qualquer área do conhecimento é um campo complexo de estudo que exige uma variedade de capacidades de seus profissionais. Dessa forma, diversas metodologias de ensino são utilizadas com o objetivo de disseminar conhecimento nos diferentes domínios que compõe uma área específica do conhecimento, visando, principalmente, o desenvolvimento de seus profissionais. A educação suportada por tecnologias da informação é uma das metodologias de ensino que vem ganhando espaço e estabelecendo um novo paradigma nesta importante área da atividade humana [Souza, Monteiro e Costa 2012].

Neste contexto, a aplicação de técnicas de Representação do Conhecimento permite atender as várias necessidades de um processo de aprendizagem, como fornecer ao computador a capacidade de raciocinar e interagir com um estudante, representando a figura de um tutor capaz de responder dúvidas ou pesquisas; e proporcionar a estruturação 
V Congresso Brasileiro de Informática na Educação (CBIE 2016)

Anais do XXVII Simpósio Brasileiro de Informática na Educação (SBIE 2016)

padronizada de informações para promover a reutilização e recuperação de forma inteligente, o que fornece ao computador a capacidade de retornar para um estudante somente aquilo que é relevante para seus estudos [Bittencourt et al. 2007].

Segundo Hoyt (2009), uma das definições da Informática em Saúde está relacionada à aplicação de tecnologias da informação em todos os campos da medicina, entre eles encontra-se a educação médica que compõe os pilares da medicina junto com a assistência e pesquisa médica.

No contexto da Informática em Saúde, ontologias são técnicas de Representação do Conhecimento que fornecem subsídios para recuperação, interpretação, gerenciamento, padronização e compartilhamento de informações que representam o conhecimento médico, além de permitir a construção de bases de conhecimento por especialistas do domínio e seu compartilhamento e reutilização por outras instituições. Porém, o potencial das ontologias não é utilizado de forma expressiva no domínio da educação em saúde suportada por tecnologia [Mota et al. 2014].

Diante do cenário apresentado, o principal enfoque deste trabalho é expor uma visão mais específica da Informática em Saúde que tem se dedicado ao desenvolvimento de diferentes softwares, abordagens e métodos computacionais com propósitos educacionais, dos quais muitos utilizam ontologia, como técnica de Representação do Conhecimento, para a modelagem e descrição semântica de conceitos de um determinado domínio da área da saúde. Para isto, foi realizada uma análise de artigos que representam pesquisas que relacionam Ontologias, Informática em Saúde e Educação, com intuito de identificar como ontologias podem contribuir para a educação suportada por tecnologias da informação no domínio da Informática em Saúde.

\section{Ontologia como Mecanismo de Representação do Conhecimento}

Segundo Davis et al. (1992), uma representação de conhecimento cria uma referência de um mundo específico e permite raciocinar sobre ela, em vez de agir sobre o mundo. Além disso, torna informações sobre o mundo passíveis de processamento automático por máquinas e permite a descoberta de novas informações a partir de informações já conhecidas a partir do raciocínio inteligente.

Ontologia é uma técnica de Representação do Conhecimento que permite a modelagem de um domínio (mundo) a partir de conceitos relevantes para o mesmo, explicitando restrições, relacionamentos entre conceitos e afirmações sobre o significado de cada conceito do domínio. Além disso, ontologia torna informações sobre o domínio passíveis de processamento automático e permite a utilização de métodos de raciocínio com a finalidade de inferir informações implícitas na estruturação deste domínio [Gruber 1993].

Com o aumento exponencial da quantidade de informação disponível no ambiente digital, destaca-se o projeto da Web Semântica. A Web Semântica representa uma série de modelos, propostos por pesquisadores de Inteligência Artificial, com a finalidade de organizar e representar a informação na Web. A ideia central é atribuir significado (sentido) aos recursos publicados na Web, de modo que sejam perceptíveis tanto pelo humano como pelo computador [Berners-Lee 2001].

Paralelamente, um conjunto de práticas para publicação e conexão de dados estruturados na Web, conhecido como Linked Data (2016), tem como objetivo 
estabelecer links entre recursos, presentes em diferentes fontes de dados, de acordo com a relação de similaridade que existe entre eles. Dessa forma, as aplicações (máquinas) podem operar uma base de conhecimento representativa sobre um tema de interesse.

Neste contexto, uma ontologia fornece um vocabulário compartilhado que promove a interoperabilidade e facilita a comunicação de ferramentas computacionais e a associação entre várias bases de conhecimento. Além disso, os conceitos estruturados na ontologia podem ser utilizados para anotar semanticamente recursos de diferentes formatos. Anotar semanticamente significa descrever o conteúdo de um recurso de uma forma explícita e inteligível para que máquinas ou agentes de software possam inferir conhecimento além do expresso explicitamente [Dovrolis et al. 2011].

\section{Educação suportada por Tecnologias da Informação no Domínio da Saúde}

A área da saúde é uma das mais recentes áreas de aplicação das Tecnologias da Informação (TI) de uma forma generalizada e organizada. Consequentemente, tornou-se possível a adoção de softwares, abordagens e métodos computacionais, com propósitos educacionais, dirigidos à formação de profissionais nesta área [Jepsen 2003].

Os softwares educacionais, por exemplo, utilizam uma abordagem que os contextualizam no processo de ensino-aprendizagem. Simuladores, por exemplo, são baseados na abordagem do estudo de casos [Souza, Monteiro e Costa 2012]. Outro enfoque é o uso de objetos de aprendizagem, que defende a abordagem da aquisição de conhecimento a partir da disponibilização de materiais didáticos com intuito de capacitar um profissional de saúde. Cada objeto de aprendizagem é um recurso digital ou não digital que representa uma unidade de ensino (vídeos, slides, etc.) utilizada, reutilizada ou referenciada durante o aprendizado suportado por tecnologias [Alvino et al. 2009].

É de fácil observação que, na área da saúde, dependendo do propósito para o qual ele foi criado, um método, abordagem ou software educacional pode expandir, complementar e melhorar a disponibilização de informação da área médica através de recursos especializados disponíveis na Web; a implementação da Educação à Distância a partir da simulação de praticamente qualquer situação que ocorra em um contexto médico educacional; a experiência clínica através de simulações de manejo de pacientes; e a educação médica continuada, ou seja, o estudo contínuo dos médicos para se manterem atualizados diante do constante progresso da medicina [Jepsen 2003].

Embora a utilização de softwares, métodos ou abordagens computacionais não seja bem aceita em algumas áreas da educação médica, a inserção de tecnologias com intuito educacional surge como um conjunto de possibilidades para elevar o nível de conhecimento e atualização de profissionais da saúde da área assistencial, educação e pesquisa, bem como para auxiliar na formação de ingressantes [Schulz e Klar 1999].

\section{Metodologia}

A metodologia de pesquisa bibliográfica utilizada foi a revisão narrativa. Este tipo de revisão descreve ou discute o desenvolvimento ou "estado da arte" de um determinado assunto, sob o ponto de vista teórico ou contextual. Apesar de não permitir a reprodução de dados e nem fornecer respostas quantitativas para questões específicas, a revisão narrativa tem papel fundamental para a educação continuada por permitir a aquisição e atualização do conhecimento sobre uma temática específica em curto espaço de tempo [Rother 2007]. 
Dessa forma, a revisão narrativa é constituída a partir de uma análise da literatura acompanhada da interpretação e análise crítica sobre o tema. Portanto, os artigos que compõe a análise desenvolvida neste estudo foram encontrados em revistas, congressos, conferências e workshops de renome e abordam os temas de interesse deste trabalho. Cada artigo utiliza ontologias como suporte para a construção de ambientes de aprendizagem que produzem conteúdos de diferentes domínios da Saúde com objetivos educacionais, mas podem divergir em dois quesitos:

1. Propósitos educacionais;

2. A contribuição da ontologia.

A análise destes dois quesitos permite a identificação das principais diretrizes das pesquisas que estudam Informática em Saúde sob a perspectiva da educação, sendo ainda possível compreender como e para quais propósitos uma ontologia é inserida neste cenário.

\section{Análise de Artigos que relacionam Ontologias, Educação e Informática em Saúde}

Dovrolis et al. (2011) e Nakikj e Weng (2013) visam disponibilizar objetos de aprendizagem da área médica na Web Semântica emergente e, consequentemente, permitir pesquisas e recuperação de objetos relevantes em bancos de dados médicos e biológicos. Para isto, ontologias são usadas para sugerir termos padronizados e conceitos no processo de criação de metadados (descrição semântica) de cada objeto de aprendizagem, para facilitar a recuperação e vinculação com outras fontes de informações da Web. O trabalho de Dovrolis et al. (2011) utiliza ontologias do domínio da saúde, e o trabalho de Nakikj e Weng (2013) estende uma ontologia, que representa dados sobre pesquisas científicas e objetos de aprendizagem, para descrever, semanticamente, objetos de aprendizagem da área da saúde. No caso específico de Nakikj e Weng (2013), são utilizados os princípios do Linked Data para a vinculação com fontes externas.

Dias et al. (2011) e Bogdan (2011) exploram os benefícios do uso de ambientes de realidade virtual imersiva e interativa relacionados com tecnologias da Web Semântica para o ensino na área de Odontologia. O papel da ontologia, criada no escopo de cada artigo, é definir conceitos (por exemplo, dente, raiz, gengiva, esmalte) que serão utilizados para a anotação de informações multimídia e modelos virtuais 3D relacionados à odontologia. Facilitando, dessa forma, a associação de modelos virtuais e informações multimídia que abordam conteúdos similares. Sendo assim, quando um aluno de odontologia está manuseando um modelo 3D (por exemplo, um dente) para realizar algum procedimento (por exemplo, extração), ele pode ter acesso ao conteúdo multimídia (por exemplo, um vídeo) que apresenta mais detalhes sobre tal procedimento.

Considerando um sistema que proporciona a reunião de médicos, que estão geograficamente distantes e são especializados em Odontologia, para discutir casos clínicos, Falkman et al. (2008) propõem um componente do sistema responsável por adicionar casos de uma forma estruturada, indicando casos relevantes para as reuniões e permitindo a pesquisa e recuperação de casos que já foram discutidos anteriormente. Estas reuniões permitem que médicos especializados e mais experientes compartilhem e disseminem seus conhecimentos, o que acaba se tornando um meio de aprendizagem para médicos menos experientes. A ontologia, neste caso, descreve dados da comunidade, composta por médicos especializados em Odontologia, e conhecimentos da área de 
V Congresso Brasileiro de Informática na Educação (CBIE 2016)

Anais do XXVII Simpósio Brasileiro de Informática na Educação (SBIE 2016)

Odontologia. Dessa forma, os casos clínicos podem ser anotados de acordo com os conceitos da ontologia, permitindo que sejam escolhidos os casos que mais se relacionam com o tema da reunião corrente.

Gao et al. (2006) desenvolvem uma infraestrutura de conhecimento científico com objetivo de reunir estudos, dados e informações sobre doenças neurodegenerativas para auxiliar os pesquisadores na busca da cura da doença de Alzheimer. Esta iniciativa permite que diversos pesquisadores tenham acesso a trabalhos e pesquisas de outros pesquisadores da mesma área, permitindo uma ampla visão e desenvolvimento do estado da arte. Neste contexto, uma ontologia, criada para subsidiar a proposta do artigo, é utilizada para organizar e descrever recursos científicos, além de promover a ligação com outros recursos. Por sua vez, ontologias do domínio da saúde são utilizadas para sugerir termos médicos para anotar o conteúdo intelectual dos recursos científicos em questão.

Boulos et al. (2006) criam um ambiente para motivar pacientes diabéticos com objetos de aprendizagem sobre a importância da medição regular da glicose e outros aspectos da diabetes (adaptados para atender às necessidades individuais do paciente). Para gerir o conhecimento necessário para a gestão da diabetes, uma ontologia fisiopatológica traça o perfil do paciente ao ser instanciada com informações relacionadas ao paciente, sendo possível, dessa forma, inferir suas necessidades e selecionar objetos de aprendizagem adequados para respondê-las.

Também com o intuito de fornecer para o paciente mais informações sobre sua doença, o trabalho de Al-busaidi, Gray e Fiddian (2006) propõe um sistema de informação que gera explicações na forma de hipertexto para pacientes com base nos dados de seus registros médicos. O sistema conta com uma ontologia, do domínio da saúde, responsável por representar o conhecimento extraído de dados médicos do paciente (diagnóstico, tratamento, etc.) e construir relações conceituais entre termos sinônimos e hierarquias. $\mathrm{O}$ objetivo da ontologia é simplificar termos médicos complexos e estender a pesquisa sobre a doença de um paciente com termos semelhantes ou relacionados, para alcançar informações relacionadas à doença, disponíveis na Web, e apresentá-las de forma simplificada, personalizada e controlada.

Com o objetivo de aumentar o tamanho da coorte (conjunto de pessoas) com doenças complexas para contribuir com pesquisas acadêmicas, o trabalho de Pathak, Kiefer e Chute (2012) busca aplicar tecnologias emergentes da Web Semântica em dados clínicos armazenados em Registros Eletrônicos de Saúde, para identificar indivíduos com doenças específicas. Neste trabalho, a função da ontologia, que é do domínio da saúde, é construir grafos virtuais a partir de dados presentes em banco de dados relacionais, ligando aqueles que possuem similaridade ou se relacionam quanto ao seu conteúdo.

Alvino et al. (2009) e Carmody, Sherry e Cardiff (2010) utilizam o modelo de sistemas de Educação à Distância, que são baseados no conceito de objetos de aprendizagem, para treinamento Médico. $O$ foco do componente educacional, apresentado em cada artigo, é verificar as deficiências de um profissional de saúde em relação às competências exigidas pelo cargo que ele ocupa e fornecer objetos de aprendizagem que o auxiliam a amenizar/eliminar essas deficiências. Neste caso em específico, o papel da ontologia é representar o domínio de Cargos e Competências e permitir inferências para identificar as lacunas existentes entre as competências dos profissionais e as competências exigidas pelas profissões que eles ocupam, facilitando a criação de planos de aprendizado personalizados e auxiliando o departamento de recursos 
V Congresso Brasileiro de Informática na Educação (CBIE 2016)

Anais do XXVII Simpósio Brasileiro de Informática na Educação (SBIE 2016)

humanos no desenvolvimento de planos de treinamento. Em particular, no trabalho de Alvino et al. (2009), a ontologia também é responsável pela anotação de objetos de aprendizagem para fornecer suporte ao processo de recomendação de objetos de aprendizagem. É importante destacar que a ontologia apresentada no trabalho de Alvino et al. (2009) é destinada para a formação/treinamento de profissionais de diferentes campos de conhecimento, inclusive da área da saúde. A ontologia apresentada em Carmody, Sherry e Cardiff (2010), por sua vez, é específica para a formação/treinamento de profissionais do domínio da saúde.

O trabalho de Evangelia et al. (2011) propõe um esquema de descrição de metadados e um repositório compatível com os requisitos do Linked Data para anotar e armazenar objetos de aprendizagem do domínio da saúde, respectivamente. Os padrões e modelos de metadados existentes no campo de aprendizagem são utilizados com a finalidade de descobrir, recuperar, compartilhar e reutilizar objetos de aprendizagem médicos de referência, e o repositório é compatível com os princípios do Linked Data para simplificar a interoperabilidade com ambientes externos. Dessa forma, ontologias do domínio da saúde são utilizadas como suporte para sugerir termos padronizados e conceitos no processo de criação de metadados de cada objeto de aprendizagem.

Blaum et al. (2013) descrevem três ontologias utilizadas na área de educação na saúde. A ontologia TIME (Topics for Indexing Medical Education), METRO (Medical Education Taxonomy Research Organization e OBEO (Ontology of Bio-Medical Educational Objectives) foram projetadas para a anotação de métodos e processos da educação médica e de objetos de aprendizagem que auxiliam na formação médica.

O objetivo do trabalho de Roth et al. (2013) é o gerenciamento de grandes quantidades de informações disponíveis em textos clínicos. Os autores propõem uma ferramenta de consulta para permitir o acesso a estes dados, em benefício de médicos e pesquisadores. Dessa forma, um vocabulário pré-definido por ontologias do domínio da saúde é utilizado para anotar semanticamente os textos clínicos.

O trabalho de Bittencourt et al. (2007) refere-se à área da Informática em Saúde para sistemas tutores inteligentes. Neste caso, a ontologia é utilizada como um formalismo de representação do conhecimento do domínio da aplicação. O enfoque do projeto é o estudo das estruturas ósseo-cranianas, atendendo a demanda de educadores da área médica interessados no ensino da anatomia e fisiologia dessas estruturas.

A proposta de Dafti et al. (2015) é a recuperação e reutilização de pacientes virtuais. Pacientes virtuais são simulações interativas de computador utilizadas como atividades de aprendizagem no ensino de cuidados de saúde. Neste trabalho, ontologias do domínio da saúde são utilizadas para a anotação semântica dos pacientes virtuais, de forma a distingui-los de outros tipos objetos de aprendizagem.

Por fim, com objetivo de propor um ambiente de aprendizagem baseado na metodologia de estudo de caso, o trabalho de Bratsas et al. (2007) tem como objetivo disponibilizar módulos de estudo formados por casos clínicos e suas soluções correspondentes. Para isto, utiliza ontologias do domínio da saúde para anotação semântica de casos clínicos, o que possibilita a recuperação e a criação de relações entre diferentes casos clínicos que retratam o mesmo problema.

As Tabelas 1 e 2 apresentam os artigos, discutidos anteriormente, organizados pelos propósitos educacionais e pela função que a ontologia exerce no contexto de cada 
V Congresso Brasileiro de Informática na Educação (CBIE 2016)

Anais do XXVII Simpósio Brasileiro de Informática na Educação (SBIE 2016)

um deles. Por uma questão de organização, os propósitos educacionais $\left(P_{i}\right)$ e funções das ontologias $\left(F_{i}\right)$ são mencionados abaixo das Tabelas 1 e 2 , respectivamente.

Tabela 1. Categorização de artigos baseada nos propósitos educacionais

\begin{tabular}{|c|c|c|c|c|c|c|}
\hline \multirow[t]{2}{*}{ Artigo } & \multicolumn{6}{|c|}{ Propósito Educacional } \\
\hline & $\mathrm{P} 1$ & $\mathrm{P} 2$ & P3 & P4 & P5 & P6 \\
\hline [Dovrolis et al. 2011] & & $\mathrm{x}$ & & & & $\mathrm{x}$ \\
\hline [Nakikj e Weng 2013] & $\mathrm{x}$ & $\mathrm{x}$ & & & & \\
\hline [Dias et al. 2011] & & $\mathrm{x}$ & & & $\mathrm{x}$ & \\
\hline [Bogdan 2011] & & $\mathrm{X}$ & & & $\mathrm{x}$ & \\
\hline [Falkman et al. 2008] & & $\mathrm{x}$ & & & & \\
\hline [Gao et al. 2006] & $\mathrm{x}$ & $\mathrm{x}$ & & & & \\
\hline [Boulos et al. 2006] & & $\mathrm{x}$ & $\mathrm{x}$ & & & \\
\hline [Al-busaidi, Gray e Fiddian 2006] & & $\mathrm{x}$ & $\mathrm{x}$ & & & \\
\hline [Pathak, Kiefer e Chute 2012] & & $\mathrm{x}$ & & & & $\mathrm{x}$ \\
\hline [Carmody, Sherry e Cardiff 2010] & & $\mathrm{x}$ & & $\mathrm{x}$ & & \\
\hline [Alvino et al. 2009] & & $\mathrm{x}$ & & $\mathrm{x}$ & & \\
\hline [Evangelia et al. 2011] & & $\mathrm{x}$ & & & & $\mathrm{x}$ \\
\hline [Blaum et al. 2013] & & $\mathrm{x}$ & & $\mathrm{x}$ & & $\mathrm{x}$ \\
\hline [Roth et al. 2013] & $\mathrm{x}$ & $\mathrm{x}$ & & & & $\mathrm{x}$ \\
\hline [Bittencourt et al. 2007] & & $\mathrm{x}$ & & & $\mathrm{x}$ & \\
\hline [Bratsas et al. 2007] & & $\mathrm{x}$ & & & & $\mathrm{x}$ \\
\hline [Dafti et al. 2015] & & $\mathrm{x}$ & & $\mathrm{x}$ & $\mathrm{x}$ & $\mathrm{x}$ \\
\hline
\end{tabular}

P1: Promover a divulgação de dados de textos clínicos ou pesquisas científicas. P2: Propagar conhecimento de áreas específicas da medicina. P3: Motivar pacientes a partir de objetos de aprendizagem que tratam da importância do uso de medicamentos. P4: Enriquecer programas de treinamento médico com objetos de aprendizagem. P5: Colaborar com propostas de ensino que utilizam tecnologias interativas. P6: Recuperar objetos de aprendizagem de referência da área médica.

Tabela 2. Categorização de artigos baseada na função da ontologia

\begin{tabular}{|c|c|c|c|}
\hline Artigo & \multicolumn{3}{|c|}{ Função da Ontologia } \\
\hline & $\mathrm{F} 1$ & $\mathrm{~F} 2$ & $\mathrm{~F} 3$ \\
\hline [Dovrolis et al. 2011] & & $\mathrm{x}$ & \\
\hline [Nakikj e Weng 2013] & $\mathrm{x}$ & & \\
\hline [Dias et al. 2011] & $\mathrm{x}$ & $\mathrm{x}$ & \\
\hline [Bogdan 2011] & $\mathrm{x}$ & $\mathrm{x}$ & \\
\hline [Falkman et al. 2008] & $\mathrm{x}$ & $\mathrm{x}$ & \\
\hline [Gao et al. 2006] & & $\mathrm{x}$ & \\
\hline [Boulos et al. 2006] & $\mathrm{x}$ & & \\
\hline [Al-busaidi, Gray e Fiddian 2006] & $\mathrm{x}$ & & $\mathrm{x}$ \\
\hline [Pathak, Kiefer e Chute 2012] & & & $\mathrm{x}$ \\
\hline [Carmody, Sherry e Cardiff 2010] & $\mathrm{x}$ & & \\
\hline [Alvino et al. 2009] & $\mathrm{x}$ & $\mathrm{x}$ & \\
\hline [Evangelia et al. 2011] & & $\mathrm{x}$ & \\
\hline [Blaum et al. 2013] & $\mathrm{x}$ & $\mathrm{x}$ & \\
\hline [Roth et al. 2013] & $\mathrm{x}$ & $\mathrm{x}$ & \\
\hline [Bittencourt et al. 2007] & $\mathrm{x}$ & & \\
\hline
\end{tabular}


V Congresso Brasileiro de Informática na Educação (CBIE 2016)

Anais do XXVII Simpósio Brasileiro de Informática na Educação (SBIE 2016)

\begin{tabular}{|c|l|l|l|}
\hline [Bratsas et al. 2007] & & $\mathrm{x}$ & \\
\hline [Dafti et al. 2015] & & $\mathrm{x}$ & \\
\hline
\end{tabular}

F1: Representar informações de áreas da medicina, dados de pesquisas científicas, dados de pacientes e profissionais da área; F2: Fornecer termos padronizados e conceitos para anotação semântica de objetos de aprendizagem; F3: Construir relações conceituais entre termos sinônimos com 0 objetivo de simplificar termos médicos complexos.

\section{Conclusão}

De uma forma geral, os artigos selecionados nesta análise estão distribuídos no intervalo de 2006 até 2015. Nota-se que no artigo de Gao et al. 2006, um dos três artigos representativos de 2006, o vocabulário que compõe a ontologia é utilizado no processo de anotação semântica de recursos digitais, visando promover a interoperabilidade semântica entre recursos de diferentes fontes. A ontologia é utilizada com os mesmos propósitos nos anos seguintes [Bratsas et al. 2007, Falkman et al. 2008, Alvino et al. 2009, Evangelia et al. 2011, Dias et al. 2011, Bogdan 2011, Dovrolis et al. 2011, Blaum et al. 2013, Roth et al. 2013 e Dafti et al. 2015]. Dessa forma, a anotação semântica de recursos digitais, que no contexto da educação são conhecidos como objetos de aprendizagem, utilizando o vocabulário estruturado de ontologias é uma prática que se mantêm em vigor devido a capacidade da ontologia de estruturar um domínio, provendo os principais conceitos, propriedades e relacionamentos.

No contexto da saúde, as vantagens da utilização de um vocabulário, padronizado e aceito por uma comunidade acadêmica, para descrever semanticamente recursos educacionais da área são a promoção da interoperabilidade de bases de conhecimento em saúde e a facilidade de recuperação, avaliação, aquisição e reutilização destes recursos. Diante disso, torna-se possível, por exemplo, a seleção de estudos sobre uma doença específica em diferentes bases médicas ou a recuperação de objetos de aprendizagem que tratam de um tema específico da área.

É importante destacar que, para descrever um domínio a partir de um vocabulário específico, a ontologia é capaz de representar conceitos sinônimos e estabelecer uma relação de equivalência entre estes conceitos. O trabalho de Al-busaidi, Gray e Fiddian (2006) utilizou esta capacidade para simplificar termos médicos complexos para a educação de pacientes com letramento em saúde inadequado. Posteriormente, o trabalho de Pathak, Kiefer e Chute (2012) também utilizaria esta capacidade para encontrar padrões em dados clínicos com a finalidade contribuir com pesquisas acadêmicas.

Finalmente, tendo em vista a eminente necessidade de gerenciamento de conhecimento por parte de diferentes softwares, abordagens e métodos computacionais com propósitos educacionais da área da saúde, os trabalhos de Boulos et al. (2006), Albusaidi, Gray e Fiddian (2006), Bittencourt et al. (2007), Falkman et al. (2008), Alvino et al. (2009), Carmody, Sherry e Cardiff (2010), Dias et al. (2011), Bogdan (2011), Blaum et al. (2013), Roth et al. (2013) e Nakikj e Weng (2013) utilizam ontologias para representar informações de áreas da medicina, dados de pesquisas científicas, dados de pacientes ou de profissionais da área. A ideia é fornecer uma camada inteligente para que softwares, abordagens e métodos computacionais com propósitos educacionais sejam capazes, por exemplo, de fornecer informações sobre uma área específica da saúde ou de conhecer o perfil e as necessidades do seu público alvo. Esta abordagem também é bastante utilizada no contexto da educação suportada por tecnologia em outras áreas do 
V Congresso Brasileiro de Informática na Educação (CBIE 2016)

Anais do XXVII Simpósio Brasileiro de Informática na Educação (SBIE 2016)

conhecimento. Bremgartner, Netto e Menezes (2015), por exemplo, utilizam ontologias em conjunto com agentes inteligentes para subsidiar o ensino flexível para alunos em Ambientes Virtuais de Aprendizagem (AVAs).

Portanto, pode-se concluir que, com aumento exponencial da quantidade de informação relacionada à saúde, disponível no ambiente digital, a utilização de ontologias é um dos métodos adequados para representação e organização de recursos digitais destinados à educação no domínio da saúde, e acredita-se que este estudo possa fornecer uma visão de como empregá-las neste contexto.

\section{Referências}

Al-busaidi, A., Gray, A. and Fiddian, N. (2006). Personalizing web information for patients: linking patient medical data with the web via a patient personal knowledge base. In Health Informatics Journal, pages 27-39.

Alvino, S. et al. (2009) "Sharing Digital Resources in Teacher Education: an Ontologybased Approach", In: SWEL Workshop on Ontologies and Social Semantic Web for Intelligent Educational Systems, Brighton, pages 97 - 105.

Berners-Lee, T., Hendler, J. and Lassila, O. (2001). The semantic web. In Scientific American Magazine, pages 34-43, v. 5, n. 284.

Bittencourt, I. I. et al. (2007) "Um Sistema Tutor Baseado em Agentes no Domínio da Medicina". In: XXVII Congresso da Sociedade Brasileira de Computação (CSBC), Rio de Janeiro, p. 372 - 379.

Blaum, W. E. et al. (2013) "Towards Web 3.0: Taxonomies and ontologies for medical education - a systematic review. In GMS Journal for Medical Education, v.30, n.1.

Bogdan, C. M. (2011) Domain Ontology of the VirDenT System. In International Journal Of Computers Communications \& Control, pages 45-52.

Boulos, M. N. K. et al. (2006). A proposed semantic framework for diabetes education content management, customisation and delivery within the M2DM project. In Computer Methods and Programs in Biomedicine, pages 188-197, London.

Bratsas, C. et al. (2007) "An Ontology-Based Approach to Constructing Medical Computational Problems for Use in Electronic Medical Education" In: ComputerBased Medical Systems, Maribor, p. 669 - 674.

Bremgartner, V., Netto, J. F. M. and Menezes C. (2015) "Explorando Arquiteturas Pedagógicas Recomendadas por meio de Agentes e Ontologia de Modelo do Aluno em Ambientes Virtuais de Aprendizagem". In: XXVI Simpósio Brasileiro de Informática na Educação (SBIE 2015), Alagoas, p. 1157 - 1166.

Carmody, L., Sherry, E. and Cardiff, J. (2010) "Healthcare Professional Roles: The Ontology Model for E-Learning”, In: ILTA Conference, Athlone, Athlone: Digital Commons Bepress, p. 1 - 8.

Dafti, E. et al. (2015) "Virtual Patients on the Semantic Web: A Proof-of-Application Study". In: Journal of Medical Internet Research, v.17, n. 1.

Davis, H. et al. (1992) "Towards an Integrated Information Environment with Open Hypermedia Systems”, In: ACM Conference on Hypertext, Milan, ACM, p. 181 - 190. 
V Congresso Brasileiro de Informática na Educação (CBIE 2016)

Anais do XXVII Simpósio Brasileiro de Informática na Educação (SBIE 2016)

Dias, D. R. C. et al. (2011) “3D Semantic Models for Dental Education” In: ENTERprise Information Systems, Springer Berlin Heidelberg, p. 89 - 96.

Dovrolis, N. et al. (2011) "Semantic Annotation and Linking of Medical Educational Resources”, In: European IFMBE Conference, Hungary, Springer, p. 1400 - 1403.

Evangelia, M. et al. (2011) "Connecting medical educational resources to the Linked Data cloud: the mEducator RDF Schema, store and API", In: Linked Learning 2011: 1st International Workshop on eLearning Approaches for the Linked Data Age, Heraklion.

Falkman, G. et al. (2008). SOMWeb: A Semantic Web-Based System for Supporting Collaboration of Distributed Medical Communities of Practice. In Journal of Medical Internet Research, Oradea, pages 1-8.

Gao, Y. et al. (2006). SWAN: A distributed knowledge infrastructure for Alzheimer disease research. In Journal of Web Semantics, Cambridge, pages 222-228.

Gruber, T. R (1993). A translation approach to portable ontology specifications. In Knowledge Acquisition, USA, v. 5, n. 2, pages 199-220.

Hoyt, R. E. et al. (2009), Medical Informatics: Practical Guide for the Healthcare Professional, Florida, Lulu.com, $3^{\text {th }}$ edition.

Jepsen, T. (2003). IT in healthcare: progress report. In It Professional, v. 5, n. 1, pages 814. Institute of Electrical \& Electronics Engineers (IEEE).

Linked Data. “The Linking Open Data cloud diagram”, http://lod-cloud.net/,Janeiro.

Mota, M. R. A. et al. (2014) "Um Mapeamento Sistemático sobre o Uso de Ontologias em Informática Médica”. In: XIV Congresso Brasileiro em Informática em Saúde (CBIS), Santos.

Nakikj, D. and Weng, C. (2013) "Extending VIVO Ontology to Represent Research and Educational Resources in an Academic Biomedical Informatics Department", In: MEDINFO 2013, Copenhagen, IOS Press, p. 1206 - 1206.

Pathak, J., Kiefer, R. C. and Chute, C. G. (2012) "Using Semantic Web Technologies for Cohort Identification from Electronic Health Records for Clinical Research", In: AMIA Summits on Translational Science, São Francisco, American Medical Informatics Association, p. 10 - 19.

Roth, C. et al. (2013) "DEDUCE Clinical Text: An Ontology-based Module to Support Self-Service Clinical Notes Exploration and Cohort Development", In: AMIA Summits on Translational Science Proceedings, North Carolina, American Medical Informatics Association, p. 1 - 3.

Rother, E. T. (2007) “Revisão Sistemática X Revisão Narrativa”. In: Acta Paulista de Enfermagem, São Paulo, v.20, n. 2.

Schulz, S. and Klar, R. (1999). Educação em Informática em Saúde. In Informática Médica, Campinas, v. 2, n. 1.

Souza, D. S., Monteiro, A. M. V. and Costa, R. M. E. M. (2012) "Sistema de Simulação de Casos para treinamento médico no domínio da Tuberculose" In: Workshop de Informática Médica. XXX Congresso da Sociedade Brasileira de Computação, Curitiba, Sociedade Brasileira de Computação, v.1. 